\title{
A Systematic Literature Review of Modern Software Visualization
}

\author{
Noptanit Chotisarn - Leonel Merino · Xu Zheng · Supaporn \\ Lonapalawong · Tianye Zhang · Mingliang Xu · Wei Chen
}

Received: date / Accepted: date

\begin{abstract}
We report on the state-of-the-art of software visualization. To ensure reproducibility, we adopted the Systematic Literature Review methodology. That is, we analyzed 1440 entries from IEEE Xplore and ACM Digital Library databases. We selected 105 relevant full papers published in 2013-2019, which we classified based on the aspect of the software system that is supported (i.e., structure, behavior, and evolution). For each paper, we extracted main dimensions that characterize software visualizations, such as software engineering tasks, roles of users, information visualization techniques, and media used to display visualizations. We provide researchers in the field an overview of the state-of-the-art in software visualization and highlight research opportunities. We also help developers to identify suitable visualizations for their particular context by matching software visualizations to development concerns and concrete details to obtain available visualization tools.
\end{abstract}

Keywords Software visualization $\cdot$ Systematic literature review $\cdot$ Information visualization

\section{Introduction}

The visualization of data in multiple domains has shown to be effective in supporting users to answer complex and frequent questions (Liu et al., 2014). Software engineering is a domain that has been benefited from visualization (Diehl, 2007). In software visualization, users are usually developers, and their tasks represent development concerns. Indeed, many software visualization approaches and systems have been proposed during the last decades. These visualizations can be classified into one of three categories depending on the visualized aspect of the subject software system (Diehl, 2007): (i) structure, e.g., to analyze how the structure of the source code of mobile applications differs from traditional software systems (Minelli and Lanza, 2013b), to ease code reading (Zhu et al., 2019); (ii) behavior, e.g., to optimize the performance of large-scale parallel programs based on execution traces (Isaacs et al., 2014), to manage cloud computing systems (Xu et al., 2019); and (iii) evolution, e.g., to analyze a fine-grained code change history (Yoon et al., 2013), to explore code quality (Mumtaz et al., 2019). Despite the many proposed software visualizations that have proven to be effective to support developers in software engineering tasks, there is limited adoption of software visualizations by developers, for instance, in context of corrective maintenance and debugging (Sensalire et al., 2008).

Noptanit Chotisarn, Supaporn Lonapalawong, Tianye Zhang, Wei Chen

State Key Lab of CAD\&CG, Zhejiang University, Hangzhou, China

E-mail: chotisarn@zju.edu.cn, 11821132@zju.edu.cn, zhangtianye1026@zju.edu.cn,chenwei@cad.zju.edu.cn

Wei Chen is the corresponding author.

Leonel Merino

Visualization Research Center, University of Stuttgart, Stuttgart, Germany

E-mail: leonel.merino@visus.uni-stuttgart.de

Xu Zheng

School of Computer Science, Zhejiang University, Hangzhou, China

E-mail: xz15968526011@hotmail.com

Mingliang Xu

College of Computer Science, Zhengzhou University, Zhengzhou, China

E-mail: iexumingliang@zzu.edu.cn 
We hypothesize that visualization approaches proposed to support software engineering tasks might include particular dimensions that differ with the state-of-the-art in information visualization. Consequently, our interest is to investigate not only software concerns tackled by visualizations, but also employed visualization techniques, roles of users, and media used to display visualizations.

The goal of our investigation is twofold:

(i) to provide researchers in the field an overview of the state-of-the-art in software visualization, and highlight research opportunities; and

(ii) to help developers to identify suitable visualizations for their particular context by matching software visualizations to development concerns.

To achieve our goal, we conducted a systematic literature review of software visualization. For each proposed visualization, we identify software visualization aspects, software engineering tasks, roles of expected users, mediums used to display visualizations, employed information visualization techniques, and name's of prototypical tools.

We found that most software visualizations support tasks that involve design and implementation, and maintenance of software systems. Though we did not find visualizations proposed to support software requirements, undoubtedly, we think they exist, and they might not appeared in our results because the our search process. We observed that software visualizations frequently use multivariate visualization techniques. Amongst them, empirical methods is the most frequent technique, which is used to assist in the design of effective software visualizations. The majority of software visualizations are visualized using standard computer screens; however, we observe that several approaches that support the analysis of the structure of systems are displayed in immersive 3D environments (e.g., virtual reality). Interestingly, we found that there is an increasing number of software visualizations that are displayed in a medium different than the computer screen.

The remainder of the paper is structured as follows: Section 2 elaborates on related work; Section 3 describes the methodology that we followed to collect and select relevant studies in software visualization; Section 4 presents our results by classifying evaluations based on the adopted categories; Section 5 discusses the results in terms of our goals and limitations of our findings; and Section 6 concludes and presents future work.

\section{Related work}

Several studies that have surveyed the literature in information visualization have focused on how information and knowledge transform into interactive visual representations across multiple domains (Geisler, 1998; Yi et al., 2007; Rodrigues-Jr et al., 2015; Kumar, 2016; McNabb and Laramee, 2017). Various reviews of the literature in information visualization have classified the state-of-the-art research and applications in multiple ways. For instance, a survey (Sun et al., 2013) analyzed visual analytics that provides an analytic space, including space and time, multivariate, text, graph and network, and other, such as applications cross-visual mapping, model-based analysis, and user interactions. Moreover, in a specific field, such as machine learning (ML), it is also represented from a visual analytics perspective that classifies the relevant work in ML field into three categories: understanding, diagnosis, and refinement (Liu et al., 2017). We observe there is a study (Liu et al., 2014), which we consider particularly useful to classify proposed visualization approaches. Consequently, we adopted this classification in our study to classify software visualizations. In it, visualization approaches are classified into one of four main categories: Empirical methodologies, Interactions, Frameworks, and Applications.

Several other studies have mainly focused on software visualization. A decade ago, Storey et al. (Storey et al., 2005) proposed a framework to classify software visualizations based on human aspects of software engineering (i.e., intent, information, presentation, interaction, and effectiveness). Kienle and Muller (Kienle and Muller, 2007) identified requirements to build and evaluate tools in software visualization. They focused on visualizations for software maintenance, re-engineering, and reverse engineering. We consider these studies important for the software visualization field. In consequence, we include in our study the aspects that these studies have analyzed. More recently, Shahin et al. (Shahin et al., 2014) investigated software visualizations that support understanding software architectures of large and complex systems, and classified visualization techniques used to support software architectures. Beck et al. (Beck et al., 2017a) conducted a survey on dynamic graph visualization, and analyzed the use of dynamic graphs to visualize software systems. They found that dynamic graph approaches have been customized to various application scenarios, one of them is software engineering. Mattila et al. (Mattila et al., 2016) presented an overview of recent software visualizations introduced in the literature. They found that only a few software visualization papers address 
software processes. Greene et al. (Greene et al., 2017) proposed a tag could-based visualization for the analysis of software project repositories. The tags visualization example was mentioned in the guest editor of the special section on software visualization by Bergel et al. (Bergel and Beck, 2017). In our study, we do not only analyze software processes, but we also analyze visualization techniques, and media used to display visualizations. Merino et al. (Merino et al., 2016, 2018b) studied fairly similar development concerns. However, there are two fundamental differences to our investigation: 1) inclusion criteria, and 2) classification method. That is, they included papers from only two venues (i.e., SOFTVIS and VISSOFT) and published until 2016, and employed a classification of visualization techniques that we think is less applicable to software visualization as the one we chose. Maletic et al. (Maletic et al., 2002) elaborates on a discussion concerning a taxonomy of software visualization related.

\section{Methodology}

We apply the systematic literature review (SLR) approach that ensures a rigorous research methodology for evidencebased software engineering. We follow Kitchenham's guidelines (Kitchenham et al., 2002) to mitigate bias in the results of the literature survey. We select this method because it offers a means for evaluating and interpreting relevant research to a topic of interest by evidence, which is robust and transferable. We apply the method by defining a review protocol that ensures rigor and reproducibility. We determine (i) data sources and search strategy, (ii) inclusion and exclusion criteria, (iii) quality assessment, (iv) data extraction, and (v) selected studies. An overview of the selection process is shown in Figure 1.

\begin{tabular}{|c|c|c|c|c|c|}
\hline Inclusion Criteria & \multirow[b]{2}{*}{$\begin{array}{l}{[\mathrm{N}=1008]} \\
{[\mathrm{N}=432]}\end{array}$} & Exclusion Criteria & \multirow[b]{2}{*}{$\begin{array}{l}{[N=841]} \\
{[N=402]}\end{array}$} & Quality Assessment & \multirow{3}{*}{$\begin{array}{l}{[\mathrm{N}=32]} \\
{[\mathrm{N}=32]} \\
{[\mathrm{N}=26]} \\
{[\mathrm{N}=2]}\end{array}$} \\
\hline \multirow[t]{3}{*}{$\begin{array}{l}\text { IEEE Xplore } \\
\text { ACM DL }\end{array}$} & & \multirow[t]{3}{*}{$\begin{array}{l}\text { Published in } 1977-2012 \\
\text { Paper length < } 8 \text { pages }\end{array}$} & & \multirow{2}{*}{$\begin{array}{l}\text { Information Visualization } \\
\text { Not a System paper } \\
\text { Paper not written in English } \\
\text { Repeated }\end{array}$} & \\
\hline & $\mathrm{N}=1440$ & & $\mathrm{~N}=197$ & & \\
\hline & & & & & $\mathrm{N}=105$ \\
\hline
\end{tabular}

Fig. 1: Stages of the search process and number of selected studies at each stage.

3.1 Data sources and search strategy

Amongst popular digital libraries and search engines that are available to collect papers, we select the ACM Digital Library $^{1}$ and the IEEE Xplore ${ }^{2}$. We select them because they have indexed the proceedings of the VISSOFT conference, which is especially dedicated to software visualization. Moreover, they also contain relevant papers from higher ranked venues, e.g., TVCG, ICPC, ICSE, VINCI, ASWEC, ICSME.

\subsection{Inclusion and exclusion criteria}

We define a search query for retrieving relevant papers in software visualization as follows:

$$
\text { "software visualization" OR "software visualisation" }
$$

We execute the query in ACM Digital Library and obtained 432 records $^{3}$. Similarly, we execute the query in IEEE Xplore and obtained 1008 records. We then exclude papers that might not be relevant in the scope of our investigation. In particular, from the 1440 records returned by the searched digital libraries, we exclude 1243 papers that corresponded to: (i) 841 papers published between 1977 and 2012, and (ii) 402 papers of less than 8 pages.

\footnotetext{
1 http://dl.acm.org/

2 http://ieeexplore.ieee.org

3 Last visit, Mar. 27, 2019
} 


\subsection{Quality assessment}

We then assess the quality of the remaining 161 papers. First, we identify 26 papers that are not written in English, which we exclude. Although we specify keywords that target specifically software visualization, we observe that some papers returned by the engines relate to visualizations proposed to support concerns in other domains. Consequently, we exclude 32 of such papers, which relate to information visualization. Next, to identified relevant software visualization papers that describe systems (from which we can extract characteristics of employed visualization techniques and the software concern that is supported), we classify the studies according to the categories proposed by Munzner (Munzner, 2008). In it, a visualization paper can be classified into one of five categories: system, technique, evaluation, design study, and model.

We select system papers as they focus on architectural choices made in the design of an infrastructure, a framework, or a toolkit, as well as, focus on lessons learned from building a system and observing its use. In the rest of the paper, we simply call them as system papers.

For each paper, we read a title, an abstract, and a conclusion. In the cases where we still were not sure of the contributions, we scan through the sections of the rest of the paper. Although papers usually exhibit characteristics of more than one type, we classify the papers by focusing on their primary contribution. Consequently, we exclude 32 papers that do not describe systems (e.g., literature reviews). When we find papers that address similar research questions that ours, for example, the work of Sensalire et al. (Sensalire et al., 2008), we discuss them into the related work.

\subsection{Data extraction}

We extract six items from each selected paper. Those are:

(i) Software aspects: we extract aspects of the software system that is visualized: structure, behavior, and evolution.

(ii) Software processes: we extract processes based on five categories: software requirements, software design and implementation, software validation, software maintenance, and all software processes.

(iii) Software engineering roles: we extract roles played by stakeholders in software engineering, who are the target users of software visualizations.

(iv) Information visualization techniques: we extract visualization techniques based on four categories:empirical methodologies, interaction, systems and frameworks, and applications.

(v) Display mediums: we extract the medium used to render a software visualization.

(vi) Tools: we extract a tool's name, and URL.

\subsection{Selected studies}

We include in our study 105 papers, of which, 57 papers have been published in VISSOFT. The remaining 48 papers have been published across 38 other venues. In Table 1, we present the list of the venues, rank, and number of papers. We searched the rankings of involved conferences and journals using two sources: CORE Rankings Portal ${ }^{4}$ and Conference Ranks ${ }^{5}$. In the former, we used the "Excellence in Research in Australia" (ERA) database (i.e., A [highest] to $\mathrm{C}$ [lowest]), and in the latter, we used the Qualis database (i.e., A1 [highest] to B5 [lowest]).

\section{Results}

We now elaborate on the results of software visualizations that we found in the research literature. We group the results based on the dimensions used in our classification. In the following, we present the results of our classifications using the six dimensions that we review (i.e., software aspects, software processes, software engineering roles, information visualization techniques, mediums, and tools). We present the results separated into two groups. Firstly, we present software engineering aspects. Those are, software aspects, software processes, and people in software engineering, which are presented in Tables 3, 4, and 5. Secondly, we present information visualization aspects, which correspond to:

\footnotetext{
4 http://www.core.edu.au/conference-portal

5 http://www.conferenceranks.com/
} 
Table 1: The list of distribution of papers by venues.

\begin{tabular}{|c|c|c|c|c|}
\hline Abbrv. & Name & Rank & Source & \# \\
\hline VISSOFT & IEEE International Working Conference on Software Visualisation & B & ERA & 57 \\
\hline TVCG & IEEE Transactions on Visualization and Computer Graphics & A & ERA & 4 \\
\hline ICPC & IEEE International Conference on Program Comprehension & $\mathrm{C}$ & ERA & 3 \\
\hline ICSE & International Conference on Software Engineering & A1 & Qualis & 3 \\
\hline VINCI & Visual Information Communications International & $\mathrm{C}$ & ERA & 3 \\
\hline ASWEC & Australian Software Engineering Conference & $\mathrm{B}$ & ERA & 2 \\
\hline ICSME & IEEE International Conference on Software Maintenance and Evolution & A & ERA & 2 \\
\hline 3PGCIC & International Conference on P2P, Parallel, Grid, Cloud and Internet Computing & B4 & Qualis & 1 \\
\hline AeroConf & IEEE International Aerospace Conference & N/A & N/A & 1 \\
\hline APSEC & Asia-Pacific Software Engineering Conference & $\mathrm{B}$ & ERA & 1 \\
\hline ASE & Automated Software Engineering Conference & A & ERA & 1 \\
\hline CASCON & Annual International Conference on Computer Science and Software Engineering & $\mathrm{B} 1$ & Qualis & 1 \\
\hline CHASE & International conference on Connected Health: Applications, Systems and Engineering Technologies & N/A & N/A & 1 \\
\hline CIT & IEEE International Conference on Computer and Information Technology & $\mathrm{C}$ & ERA & 1 \\
\hline COP & International Workshop on Context-Oriented Programming & N/A & N/A & 1 \\
\hline CSMR & European Conference on Software Maintenance and Reengineering & $\mathrm{C}$ & ERA & 1 \\
\hline EDOC & IEEE International Enterprise Distributed Object Computing Conference & B & ERA & 1 \\
\hline ENASE & International Conference on Evaluation of Novel Approaches to Software Engineering & B & ERA & 1 \\
\hline FDG & International Conference on the Foundations of Digital Games & $\mathrm{C}$ & ERA & 1 \\
\hline GECCO & Genetic and Evolutionary Computation Conference Companion & A & ERA & 1 \\
\hline ICSE-SEIP & ICSE Software Engineering In Practice & A1 & Qualis & 1 \\
\hline IEEE Access & IEEE Access & N/A & N/A & 1 \\
\hline IMWUT & Proceedings of the ACM on Interactive, Mobile, Wearable and Ubiquitous Technologies & N/A & N/A & 1 \\
\hline INCISCOS & International Conference on Information Systems and Computer Science & N/A & N/A & 1 \\
\hline IV & International Conference on Information Visualisation & B & ERA & 1 \\
\hline MCC & MCC workshop on Mobile cloud computing & N/A & N/A & 1 \\
\hline MODELSWARD & International Conference on Model-Driven Engineering and Software Development & N/A & N/A & 1 \\
\hline MSR & IEEE International Working Conference on Mining Software Repositories & A & ERA & 1 \\
\hline MTD & Workshop on Managing Technical Debt & N/A & N/A & 1 \\
\hline QRS-C & IEEE International Conference on Software Quality, Reliability and Security Companion & $\mathrm{B}$ & ERA & 1 \\
\hline ROOTS & Reversing and Offensive-oriented Trends Symposium & N/A & N/A & 1 \\
\hline SBES & Brazilian Symposium on Software Engineering & B2 & Qualis & 1 \\
\hline SCAM & IEEE International Workshop on Source Code Analysis and Manipulation & $\mathrm{C}$ & ERA & 1 \\
\hline SCCG & Spring Conference on Computer Graphics & $\mathrm{C}$ & ERA & 1 \\
\hline TPDS & IEEE Transactions on Parallel and Distributed Systems & N/A & N/A & 1 \\
\hline $\mathrm{VL} / \mathrm{HCC}$ & IEEE Symposium on Visual Language and Human-Centric Computing & $\mathrm{B}$ & ERA & 1 \\
\hline Web3D & International ACM Conference on 3D Web Graphics and Interactive Technology & B1 & Qualis & 1 \\
\hline WiPSCE & Workshop in Primary and Secondary Computing Education & N/A & N/A & 1 \\
\hline
\end{tabular}

information visualization techniques, display mediums, and tools. These aspects are presented in Tables 6,7 , and 8 . We also present exemplary software visualization tools (shown in Figures 2, 3, and 4). We select examples that exhibit the highest number of citations.

\subsection{Software Engineering Aspects}

Software development is directly related to the software process. In the software process, there are many people involved in the process who are stakeholders of the software project. Each phase in software development has multiple related software components. There are many aspects of software that project stakeholders need to understand, for which software visualization can be useful. Consequently, we discuss (i) aspects of software systems, (ii) involved software process, and (iii) roles of stakeholders involved in software engineering.

\subsubsection{Classification by software system aspects}

We classify the software visualizations based on the categories proposed by Diehl (Diehl, 2007). His taxonomy classifies software visualizations based on the aspects of software that are supported. In it, software visualizations belong to one of three categories: (i) structure, which includes visualizations that support the analysis of the static aspects and relationships in software systems, (ii) behavior, which relates to visualizations proposed for the analysis of data 


\begin{tabular}{lrrr}
\hline Year & VISSOFT & Other Venues & Total \\
\hline 2013 & 7 & 9 & 16 \\
2014 & 10 & 3 & 13 \\
2015 & 10 & 8 & 18 \\
2016 & 7 & 5 & 12 \\
2017 & 8 & 3 & 11 \\
2018 & 10 & 8 & 18 \\
2019 & 5 & 12 & 17 \\
\hline SUM & 57 & 48 & 105 \\
\hline
\end{tabular}

\begin{tabular}{lr}
\hline Software Aspects & Total \\
\hline Structure & 34 \\
Behaviour & 34 \\
Evolution & 37 \\
\hline
\end{tabular}

Table 3: Software aspects involved in software visualizations.

Table 2: Number of software visualization papers by year and venue.

\begin{tabular}{lr}
\hline Software Processes & Total \\
\hline Software design and implementation & 51 \\
Software maintenance & 24 \\
All software processes & 23 \\
Software validation & 7 \\
Software requirements & 0 \\
\hline
\end{tabular}

Table 4: Software processes supported by software visualizations.

\begin{tabular}{lr}
\hline $\begin{array}{l}\text { Information visualization tech- } \\
\text { niques }\end{array}$ & Total \\
\hline Empirical methodologies & \\
$\quad$ Model & 52 \\
$\quad$ Evaluation & 9 \\
Interactions & \\
$\quad$ WIMP interactions & 5 \\
$\quad$ Post-WIMP interactions & 5 \\
Frameworks & \\
$\quad$ Systems and frameworks & 3 \\
Applications & \\
$\quad$ Multivariate data visualization & 13 \\
$\quad$ Graph visualization & 7 \\
$\quad$ Text visualization & 6 \\
$\quad$ Map visualization & 5 \\
\hline
\end{tabular}

\begin{tabular}{lr}
\hline Roles & Total \\
\hline Developer & 63 \\
Practitioner & 11 \\
Software Engineer & 8 \\
Maintainer & 5 \\
End-user & 4 \\
Project Manager, Researcher, Tester & 3 \\
Analysts, Architect & 2 \\
Team Member & 1 \\
Not Identified & 23 \\
\hline
\end{tabular}

Table 5: Frequency of targeted roles played by users of software visualizations.

\begin{tabular}{lr}
\hline Mediums & Total \\
\hline Standard Screen & 87 \\
Immersive 3D Environment & 8 \\
Wall Display & 2 \\
Multi-Touch Table & 1 \\
Not Identified & 7 \\
\hline
\end{tabular}

Table 7: Mediums used to display software visualizations.

Table 6: Number of software visualizations grouped by information visualization techniques.

collected from the execution of programs, and (iii) evolution, which contains visualizations that support to analyze how systems change over time. Therefore, we analyze the number of papers that relate to each of these categories in the studied period.

Table 3 shows that the number of papers that we found across the three aspects is almost balanced: 37 papers focus on the visualization of software evolution, 34 papers discuss the visualization of software behavior, and 34 papers describe visualizations of software structure.

The structure of software systems can be represented using a city metaphor (e.g., buildings in the city represent classes in the software system). An example of a software visualization that uses the city metaphor is found in $V R$ City (Vincur et al., 2017) (see Figure 4a). VR City enables users to observe and interact with a visual representation of source code that is displayed in a virtual reality environment. Another study (Isaacs and Gamblin, 2018) employs a directed acyclic graph to analyze dependencies of package management systems. Ravel (Isaacs et al., 2014) allows users to visualize the behaviour of a software system during the execution of a program based on log traces. In it, time is used to understand the parallelism in the execution of a program. Ravel allows users to search the event history to unveil insights. Users interact with the visualization using a standard computer screen, and mouse and keyboard. An 


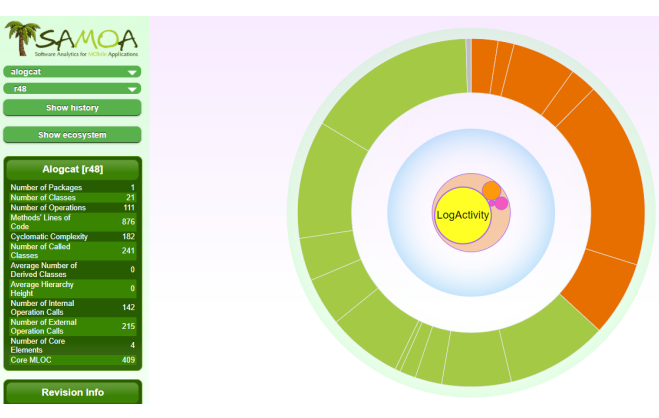

(a) SAMOA, 2013

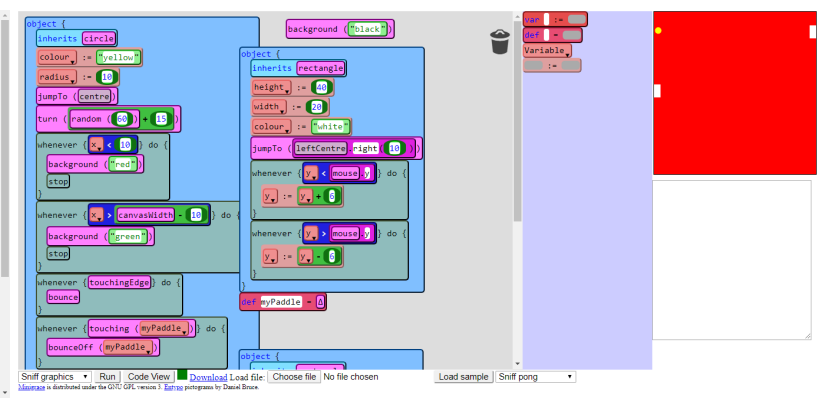

(b) Tiled Grace, 2014

Fig. 2: 2a; SAMOA displays the source code of the Alogcat application. Figure taken from the Web (Minelli and Lanza, 2013a), and reused with permission (c) 2013 Minelli. 2b; Tiled Grace editing a small program in the "sniff graphics" dialect. Figure taken from the Web (Homer and Noble, 2014b), and reused with permission ${ }^{\complement} 2014$ Homer.

example of a visualization that focus on code change history is Azurite (Yoon et al., 2013). In it, users can visualize the evolution of a software system that is displayed in a standard computer screen. Azurite is integrated into Eclipse as a plug-in. It enables developers and maintainers to go through the history of code via several editor commands.

\subsubsection{Classification by software engineering processes}

We classify selected papers by software processes based on a set of four process activities (Sommerville, 2011) that are fundamental to software engineering: (i) software specification (specifically software requirements), which deals with the specification of software functionality and its constraints definition process. (ii) software design and implementation, which translates a specification into a concrete software product. (iii) software validation or software testing, which prevents that a software system exhibits incorrect behavior. (iv) software evolution (specifically software maintenance), which deals with changes to a software system to deal with new requirements and discovered bugs that need to be fixed. We organize the papers based on keywords that describe the name of a process, the abstract set of activities, and the tools introduced in those processes.

Table 4 presents the results of our classification of papers by software process, we found: 51 papers that we classified into the design and implementation process, 24 papers that deal with the maintenance process, 23 papers that concern to all software processes, and 7 papers that focus on the software validation process. We did not find visualizations proposed to support software requirements, though we are aware of the existence of such papers. Still, we consider that our results can give an overview of the degree of importance of each category.

One study (Benomar et al., 2013) uses a heatmap visualization to represent the execution and evolution of software during implementation. The task of this heatmap tool is to explore software dynamicity based on time and other dimensions of a software system. The tool is displayed on a standard computer screen. Another study (Gouveia et al., 2013) propose three dynamic graphical forms, namely Sunburst, Vertical Partition, and Bubble Hierarchy to deal with fault detection in the verification process. The tool employs HTML5 to visualize software fault localization. The tool is offered as a plug-in for Eclipse and it is displayed on the standard computer screen. CTRAS (Hao et al., 2019) proposes a crowdsource testing method that takes advantage of code clones to enrich the content of bug descriptions and improve the efficiency of inspecting test reports through a comprehensive and comprehensible report. SAMOA (Minelli and Lanza, 2013b) (see Figure 2a) is a visualization tool that supports software maintenance tasks. The tool visualizes source code, third-party libraries, and historical data using pie charts. The tool supports in-depth analysis of structural and evolutionary aspects of systems. The tools is implemented as a web application that is displayed on the standard computer screen. One study (Fronza et al., 2013) uses a Wordle visualization to support all software processes. The visualization tool, which is displayed on the computer screen, supports tasks that deal with the cooperation level of a development team using a Wordle-like visualization technique.

\subsubsection{Classification by software engineering roles}

We extracted the roles that envisioned users of software visualizations play in software engineering from frequent keywords in papers. In Table 5 we present the results of our classification of papers by software engineering roles. We 
found that proposed visualization envisioned ten different software engineering roles for their targeted users: Developer, Practitioner, Software Engineer, Maintainer, End-user, Project Manager, Researcher, Tester, Analysts, Architect, and Team Member. Notice that the second most frequent category corresponded to papers in which we did not identify an explicit role.

Roles such as developer, maintainer, and software engineer are directly related to the software implementation process and software maintenance process (and evidently related to all software processes), which are the top five processes mostly visualized. Interestingly, we found that the practitioner is the second largest number after the developer. Students, apprentices and new workers organize the practitioner.

One study (Minelli et al., 2014) proposed a visualization, which is displayed on a standard computer screen, to analyze how developers use a graphical user interface of an integrated development environment. In it, developers need to understand and characterize development sessions with a timeline. Variability Blueprint (Urli et al., 2015), displayed on a computer screen, supports software product line (SPL) engineering through a visualization of feature models tree. The visualization tool helps software maintainers to understand dependencies between feature models. RepoGrams (Rozenberg et al., 2016) is a visualization tool for the analysis of software repositories (see Figure 3b). The tool, which is displayed on the standard computer screen, uses an extensible, metrics-based, visualization model as a footprint of the repository. The tool supports software engineers and researchers in comparative analyses of software projects over time. Performance Evolution Matrix (Sandoval Alcocer et al., 2019) is an interactive visualization that uses a matrix layout for the analysis of runtime metrics and source code changes, e.g., execution graphs. The tool supports the analysis of performance metrics at different granularity levels of multiple versions of a software system. The study elaborates on evidence from an experiment that showed benefits of such interactive visualization for practitioners.

\subsection{Information Visualization}

We characterize aspects of software visualizations based on an employed information visualization technique, mediums, and tools.

\subsubsection{Classification by information visualization techniques}

Table 6 presents our classification of the selected papers by information visualization technique based on four categories introduced in a previous study (Liu et al., 2014): (i) empirical methodologies usually correspond to novel visualization models and usability studies, (ii) interactions papers (Yi et al., 2007) provide a comprehensive survey to study the role of interaction techniques in information visualization and the study, which can be further split into two categories: WIMP and post-WIMP interactions, (iii) systems and frameworks, that is, systems refer to toolkits for visualization construction, and frameworks represent modeling of visualization techniques, and (iv) applications, in which visualization designs are split into four groups based on the characteristics of the target data.

We found 61 software visualization papers that we classified as empirical methodologies, 31 papers as applications, 10 papers into the interactions category, and 3 papers as systems and frameworks.

Empirical methodologies Information visualization researchers have developed multiple empirical methods to support the design and implementation of novel visualization techniques, which can be classified in two subcategories: model and evaluation (Liu et al., 2014). Models are the basis of empirical research. Indeed, various models have been developed to assist in the design of effective data visualization. On the other hand, there are multiple challenges when evaluating software visualizations (Merino et al., 2018a). User studies are the most common method used in evaluations of information visualizations, which often involved measuring visualization performance.

We found that amongst the 61 empirical methodologies papers, 52 papers correspond to the model category and 9 to evaluations. We observe there is an overlap between model and evaluation methods.

CuboidMatrix (Schneider et al., 2016) is a visualization that uses a space-time metaphor to support users on understanding software evolution. Thus, users can navigate the space-time visualization to solve software comprehension tasks. The visualization is presented on a standard screen.

ExploViz (Fittkau et al., 2015a) is an example of visualization evaluation (see Figure 3a). In it, a model of the architecture of a software system is visualized using a flat landscape view. In the view, (1) green boxes represent nodes in the architecture and white labels are used to display the hostname of nodes; (2) purple boxes represent applications 


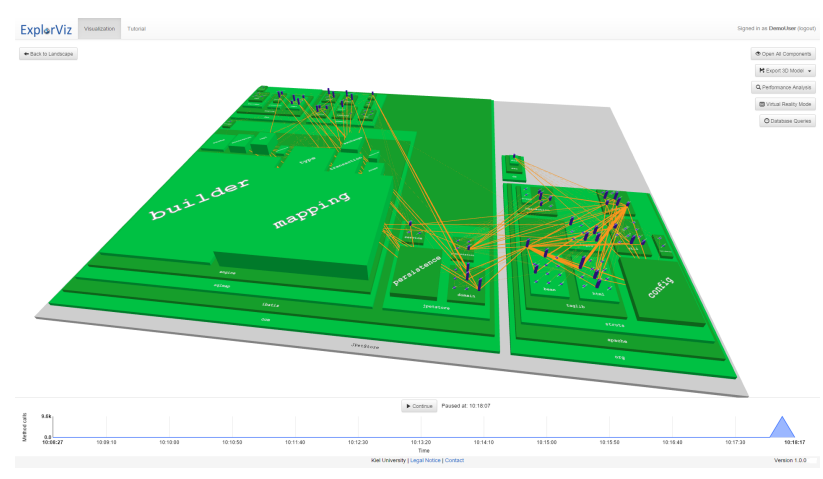

(a) ExplorViz, 2015

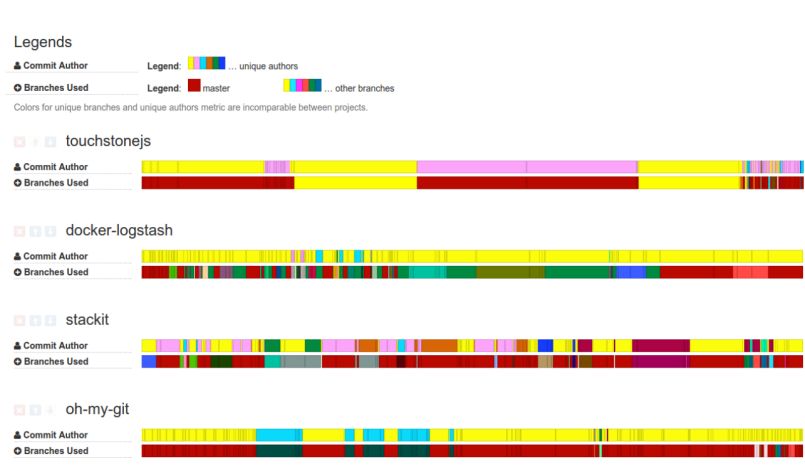

(b) RepoGrams, 2016

Fig. 3: 3a; ExploViz supports the analysis of the architecture of a software system using a flat landscape 3D application visualization. Figure taken from the Web (Fittkau et al., 2015b), and reused with permission (C) 2015 Hasselbring. 3b; the RepoGrams visualization shows eight software repository footprints, reused with permission (c) 2016 Rozenberg (Rozenberg et al., 2016).

running amongst nodes; and (3) orange lines represent messages amongst applications. The visualization is presented on a standard screen. In a subsequent investigation, authors implemented a similar visualization displayed in virtual reality using a immersive 3D environment and 3D physical printed models.

Interactions User interaction is essential for data representation and data analysis. Interaction technologies can be classified in two categories: WIMP interaction (i.e., window, icon, mouse, pointer) and Post-WIMP interactions, which is beyond traditional WIMP interaction, e.g., reality-based interaction and 3D interaction. In papers that mention various categories, we identify a main interaction technique based on the content of the paper.

Amongst the interactions reported on papers, we found that 5 correspond to WIMP and 5 to post-WIMP interactions. We observe that interactions often depend on the medium used to display visualizations.

ConceptCloud (Greene and Fischer, 2015) combines a tag cloud visualization technique with a concept lattice to support source code navigation based on a flexible and interactive browser for SVN and Git repositories. This tool is an example of WIMP interactions. Visualizations are rendered on the standard screen.

We also found a few post-WIMP interactions that often are used for visualizing software structure. VR City (Vincur et al., 2017) employs virtual reality to display a software visualization that uses a city metaphor. Figure 4a presents views of the tool in which users can compare the characteristics of two software cities: JHotDraw (top) and JUnit (bottom). We observe that tjhe JUnit project looks smaller than JHotDraw, even though, it contains many more classes. Authors provide videos to obtain further details ${ }^{6}$.

Frameworks Systems and frameworks has only one subcategory. Systems refer to libraries or toolkits for developing visualizations, whereas frameworks are used to model various aspects of a visualization technique. We found in this category only $2.86 \%$ of 105 analyzed papers (i.e., 3 papers. D3.js is probably the most popular library used to create Web interactive visualizations. Indeed, D3 is a powerful visualization framework that can be used to create interactive visualizations based on HTML5, SVG, and CSS.

CodeCompass (Porkoláb et al., 2018) is an open source LLVM/Clang-based tool developed by Ericsson Ltd. and Etvs Lornd University, Budapest. The tool uses a variation of component diagrams which are implemented as graphs. The tool aims to help users understanding large legacy software systems based on static analysis and software metrics. CodeCompass is a web-based plugin that is highly extensible.

Applications To analyze the various types of data sets that can be involved in visualization, a study (Liu et al., 2014) proposes 4 categories based on the characteristics of a data set. We found that nearly half of the papers in the applications category (i.e., 31 papers) visualize multivariate data sets (i.e., 13 papers). The rest can be split into graphs (i.e., 7 papers), texts (i.e., 6 papers), and maps (i.e., 5 papers) respectively.

\footnotetext{
6 https://goo.gl/inrcZs
} 


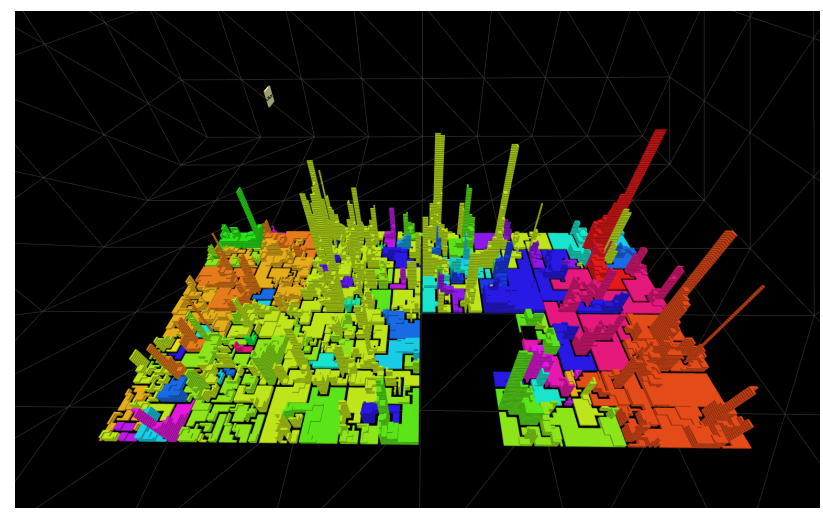

(a) VR City, 2017

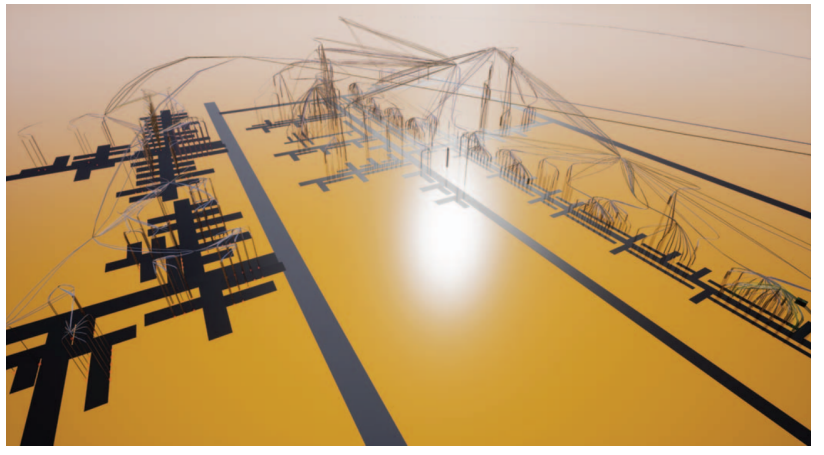

(b) EvoStreets, 2018

Fig. 4: 4a; The side view of $V R$ City, a city metaphor visualizes a software system in Virtual Reality environment, reused with permission ( 2017 Vincur (Vincur et al., 2017). 4b; The birds eye view of EvoStreets visualization, reprinted with permission ${ }^{\circledR} 2018$ Koschke (Rüdel et al., 2018).

The applications category take into account a visualization design that can involve an underlying graph, textual, map, and multivariate data. Therefore, we classified the list of the specific applications based on the underlying data and the visualization techniques. For example, one study (Barik et al., 2014) elaborates on how developers comprehend compiler messages, which is a type of textual data, by providing a notification construction. Indeed, evaluation results are often used by developers to find explanations to development concerns. We group this work into the classification of text applications and behavior as the notation visualization is part of the textual data application.

Firstly, graph-like data is related to topological structures such as dependencies amongst objects in an objectoriented systems or relationships among a group of people. Linvis (Wilde and German, 2018) is a visualization tool for the analysis of software repositories, which uses a conversion of directed acyclic graphs (DAG) to merged trees. This tool supports maintainers on the analysis of small projects. These methods and visualizations are implemented for the Web and displayed on the standard screen.

Secondly, we observe that the visualization of textual data aims at supporting the analysis of the semantics of data. For example, a visualization such that could helps software engineers to understand important concerns in an extensive collection of software project documents. An example of text visualization is the tile-based visualization named Tiled Grace (Homer and Noble, 2014a) (see Figure 2b). Using drag-and-drop, developers interact with tiles to edit their programs, which they can complement with traditional textual environments. This tool is an educational programming language with a conventional textual syntax. This visualization is displayed on the standard screen.

Thirdly, visualizations to understand geological data that relates space and size restricted to a terrestrial area. CodeSurveyor (Hawes et al., 2015) is a visualization tool that uses a cartographic metaphor for creating a map of a code base. The interactive map allows users to zoom out and obtain a high-level overview of software components in which source files are shown as states in continents. The visualization is displayed on the standard screen.

Lastly, multivariate data is a generic type that relates to a variety of fields. Usually, the goal of visualization of multivariate data is to explore the relationship amongst their dimensions. In many cases, the use of varying visualization techniques to understand inter-relationships is conducted by researchers and engineers. TraceDiff (Trumper et al., 2013) is a novel visualization method, which is based on icicle plots and hierarchical edge bundles. The approach uses a multiscale visualization metaphor to support the analysis of event traces, code structure, and function calls. The visualization is displayed on a standard screen.

\subsubsection{Classification by mediums}

Table 7 presents the medium used to render software visualizations. A previous study (Merino et al., 2018b) analyzed the research literature on software visualization to extract development tasks, visualization techniques, and in particular, the mediums, which we adopted in our study as well. We confirmed that the standard screen is the most frequent medium used to display software visualization (i.e., 87 papers). We also observe that in some papers authors do not explicitly mention the medium, though we infer it is a standard screen. Often, we infer the medium based on 
the analysis of figures in papers. Amongst the analyzed papers, we found a few approaches that used a medium other than the standard computer screen to display visualizations. In particular, we found 3D environments (i.e., 8 papers), wall displays (i.e., 2 papers), and multi-touch tables (i.e., 1 paper) respectively. We also counted the number of papers in which we did not identify an explicit medium (i.e., 7 papers).

SArF Map (Kobayashi et al., 2013) visualizes components and layers of software systems using a city metaphor combined with a generated map. Developers and non-developers can use this visualization for high-level discussions and to make decisions in software development. This visualization is displayed on a standard computer screen. EvoStreets (Rüdel et al., 2018) presents a software city metaphor visualization as well. Figure $4 \mathrm{~b}$ shows a view of the visualization. Buildings in the visualization represent methods in the system, edges represent method calls, and streets represent package nesting and classes. This visualization is displayed in a 3D immersive virtual reality environment. Chronotwigger (Ens et al., 2014) enables the visualization of source and test files as visual nodes that dynamically change over time. This multi-user collaborative software visualization tool supports understanding source code and test code co-evolution. In it, users can specify a time span and select a node and zoom-in/out to analyze co-change. This visualization is displayed on a wall display combined with an immersive 3D environment. SourceVis (Anslow et al., 2013) is a visualization tool designed to support multiple users, multiple visualization techniques, and to be displayed on a large shared interactive surface such as a multi-touch table. The aim of this tool is to understand how a system is structured based on the visualization of software metrics and source code evolution. This tool uses multi-touch tables for multi-user collaborative applications.

\subsubsection{Classification by tools}

Amongst the 105 reviewed papers, we found 62 papers in which we identified tools' names (see Table 8). That is, in only 28 papers we found an available link. When analyzing the evolution of the number of tools in the period $2013-$ 2019 , we observe that the number of tools per year fluctuate between $3(2013,2014,2017,2019)$ and $6(2016)$. We marked papers that contain available links with check mark $(\checkmark)$ (and with dash $(-)$ papers in which we did not found an available link). Moreover, tools are linked to URLs where they are available. Each tool is classified by: (i) Tool Names, (ii) References, (iii) Citation count ${ }^{7}$, (iv) Software aspects (SoftVis), (v) Software engineering process (i.e., Software requirements [RE], Software Design and implementation [DI], Software validation [VV], Software maintenance [MA], All software processes [ALL]), (vi) Software Engineering Roles (Analyst [ANL], Architect [Arch], Developer [Dev], Maintainer [MTN], Practitioner [Prac], Project Manager [PM], Researcher Res, Software Engineer [SE], Team Member [Team], Tester [Test], End-user [User], Not Identified [N/A]), (vii) Information visualization aspects (InfoVis) (viii) Medium (3D environments [3D], Multi-touch table/screen [MT], Standard screen [SS], Wall display [WL]; Not Identified [N/A]). (ix) Available code, We think our list of available software visualization tools can offer a suitable complement to previous studies (Merino et al., 2019), and represent a means for practitioners who are willing to adopt software visualizations.

\section{Discussion}

In the following section, we discuss on the results of the topics described previously, i.e., Software system aspects, Software engineering processes, Software engineering roles, Information visualization techniques, Mediums, and Tools.

Software Systems Aspects. We found that the number of software visualizations proposed to support each of the three aspects of systems (i.e., structure, behavior, and evolution) is almost balanced. We found that $32.39 \%$ (i.e., 34 papers) of the analyzed papers describe visualizations proposed for the analysis of the structure of software systems. We found that more than a third of papers, i.e., $35.24 \%$, (i.e., 37 papers) focus on visualizations of the evolution. We also found that $32.39 \%$ (i.e., 34 papers) of the 105 reviewed papers relate to visualizations proposed to analyze the behavior of software systems.

We think that visualizations proposed to deal with questions that relate to these aspects are a good fit to the increasing complexity of software systems. We expect our review can be used by practitioners to obtain an overview of modern software visualization tools, some of them available, that have been proposed in the research literature. However, if we look at the number of available tools, we observe that the lowest number of tools are present in the Behavior (i.e., 6 tools), then the Structure aspect in which we found 10 available tools, and finally, the Evolution aspect in which available tools are a bit more frequent (i.e., 12 tools).

\footnotetext{
7 Last visit, Jan. 27, 2020
} 
Table 8: The list of software visualization tools organized by publication year and citation count. A asterisk mark is used to identify a publication year that differs between Google Scholar and IEEE Xplore.

\begin{tabular}{|c|c|c|c|c|c|c|c|c|}
\hline Tool Names & References & Citation & SoftVis & Process & Roles & InfoVis & Medium & Available \\
\hline CTRAS & (Hao et al., 2019) & 3 & $\mathrm{E}$ & VV & Dev, Test & Model & SS & $\checkmark$ \\
\hline Performance evolution matrix & (Sandoval Alcocer et al., 2019) & 1 & $\mathrm{E}$ & MA & Dev, Prac & Model & SS & - \\
\hline CloneCompass & (Wang et al., 2019) & 0 & $\mathrm{E}$ & MA & SE & Model & SS & - \\
\hline CorpusVis & (Slater et al., 2019) & 0 & $\mathrm{~S}$ & MA & Dev & Model & SS & - \\
\hline Internal usage map & (Anquetil et al., 2019) & 0 & $\mathrm{~S}$ & MA & Dev & Model & SS & - \\
\hline Evo-Clocks & (Alexandru et al., 2019) & 0 & $\mathrm{E}$ & MA & Dev & Model & SS & - \\
\hline EvoStreets (extended result) & (Steinbeck et al., 2019) & 0 & $\mathrm{~S}$ & ALL & Dev, Prac & Evaluation & $3 \mathrm{D}$ & - \\
\hline Atria & (Williams et al., 2019) & 0 & $\mathrm{~B}$ & DI & Dev & Model & SS & - \\
\hline Callflow & (Nguyen et al., 2019) & 0 & $\mathrm{~B}$ & ALL & Dev, ANL & Model & SS & $\checkmark$ \\
\hline City on the river (CotR) & (Perrie et al., 2019) & 0 & $\mathrm{E}$ & ALL & Dev, Team & Model & SS & - \\
\hline Parallel & (Zhu et al., 2019) & 0 & B & DI & Prac & Model & SS & $\checkmark$ \\
\hline Clouds-Pi & (Toosi et al., 2018) & 8 & $\mathrm{~S}$ & VV & N/A & Graph & N/A & $\checkmark$ \\
\hline EvoStreets & (Rüdel et al., 2018) & 6 & $\mathrm{~S}$ & ALL & Dev, Prac & Evaluation & $3 \mathrm{D}$ & - \\
\hline Feature visualiser & (Duhoux et al., 2018) & 6 & $\mathrm{~B}$ & DI & Dev & Model & N/A & - \\
\hline CodeCompass & (Porkoláb et al., 2018) & 3 & $\mathrm{E}$ & ALL & Dev & Framework & SS & $\checkmark$ \\
\hline BuildExplorer & (Lebeuf et al., 2018) & 2 & $\mathrm{E}$ & DI & Dev, SE & Model & SS & $\checkmark$ \\
\hline RepoVis & (Feiner and Andrews, 2018) & 2 & $\mathrm{E}$ & DI & Dev, PM & Multivariate & SS & - \\
\hline Parceive & (Wilhelm et al., 2018) & 1 & $\mathrm{~B}$ & DI & Dev & Evaluation & SS & - \\
\hline Quality models inside out & (Ulan et al., 2018) & 1 & $\mathrm{E}$ & MA & N/A & Multivariate & SS & $\checkmark$ \\
\hline Remotion & (Qian et al., 2018) & 0 & $\mathrm{E}$ & ALL & ANL, User & Model & $3 \mathrm{D}$ & $\checkmark$ \\
\hline VR city & (Vincur et al., 2017) & 24 & $\mathrm{~S}$ & DI & N/A & PostWIMP & $3 \mathrm{D}$ & - \\
\hline Code park & (Khaloo et al., 2017) & 12 & $\mathrm{~S}$ & DI & Dev & WIMP & $3 \mathrm{D}$ & - \\
\hline CodeCity & (Ogami et al., 2017) & 9 & $\mathrm{~B}$ & DI & Dev & Map & SS & - \\
\hline Method execution reports & (Beck et al., 2017b) & 8 & $\mathrm{~B}$ & DI & Dev & Text & SS & $\checkmark$ \\
\hline TraceCompare & (Doray and Dagenais, 2017) & 7 & $\mathrm{~B}$ & DI & Dev & Multivariate & N/A & - \\
\hline iTraceVis & (Clark and Sharif, 2017) & 6 & $\mathrm{~S}$ & DI & Dev, Res & Model & SS & $\checkmark$ \\
\hline RegExVisualizer & (Hollmann and Hanenberg, 2017) & 3 & $\mathrm{~S}$ & DI & Prac & Evaluation & SS & $\checkmark$ \\
\hline RepoGrams & (Rozenberg et al., 2016) & 17 & $\mathrm{E}$ & MA & SE, Res & Multivariate & SS & $\checkmark$ \\
\hline WebDPF & (Rabbi et al., 2016) & 15 & $\mathrm{~S}$ & DI & N/A & Model & SS & $\checkmark$ \\
\hline Jsvee \& Kelmu & (Sirkiä, 2018)* & 12 & B & DI & Dev, Prac & Text & SS & - \\
\hline TypeV & (Feist et al., 2016) & 9 & $\mathrm{E}$ & ALL & Dev & Model & SS & $\checkmark$ \\
\hline CuboidMatrix & (Schneider et al., 2016) & 8 & $\mathrm{~B}$ & MA & Dev & Model & SS & - \\
\hline Walls, pillars and beams & (Tymchuk et al., 2016) & 4 & $\mathrm{E}$ & VV & Dev & Model & $3 \mathrm{D}$ & $\checkmark$ \\
\hline Linvis & (Wilde and German, 2018)* & 2 & $\mathrm{E}$ & MA & MTN & Graph & SS & $\checkmark$ \\
\hline Perquimans & (Middleton and Murphy-Hill, 2016) & 0 & $\mathrm{~S}$ & ALL & Prac, Res & Graph & SS & $\checkmark$ \\
\hline ExplorViz & (Fittkau et al., 2015a) & 19 & $\mathrm{~S}$ & DI & N/A & Evaluation & SS & $\checkmark$ \\
\hline ConceptCloud & (Greene and Fischer, 2015) & 12 & $\mathrm{E}$ & MA & Dev & WIMP & SS & $\checkmark$ \\
\hline CodeSurveyor & (Hawes et al., 2015) & 11 & $\mathrm{~S}$ & DI & Dev & Map & SS & - \\
\hline Developer rivers & (Burch et al., 2015) & 11 & $\mathrm{E}$ & ALL & Dev & Model & SS & - \\
\hline Variability blueprint & (Urli et al., 2015) & 10 & $\mathrm{E}$ & DI & MTN & Model & SS & - \\
\hline Vimetrik & (Khan et al., 2015) & 5 & $\mathrm{E}$ & MA & N/A & Model & SS & - \\
\hline Goldenberry-GA & (Garzón-Rodriguez et al., 2015) & 3 & $\mathrm{~S}$ & MA & N/A & Model & SS & - \\
\hline PhysViz & (Scarle and Walkinshaw, 2015) & 3 & $\mathrm{~S}$ & ALL & Dev & WIMP & SS & $\checkmark$ \\
\hline Cerebro & (Palepu and Jones, 2015) & 2 & $\mathrm{~B}$ & DI & SE & Model & SS & $\checkmark$ \\
\hline JETracer & (Molnar, 2015) & 1 & B & DI & Dev & Framework & SS & $\checkmark$ \\
\hline OctMiner & (Lessa et al., 2015) & 0 & $\mathrm{~S}$ & DI & N/A & Evaluation & SS & - \\
\hline Tiled grace & (Homer and Noble, 2014a) & 45 & $\mathrm{~B}$ & DI & Dev & Text & SS & $\checkmark$ \\
\hline Feature relations graphs (FRoGs) & (Martinez et al., 2014) & 32 & $\mathrm{E}$ & MA & N/A & Model & SS & - \\
\hline AniMatrix & (Rufiange and Melançon, 2014) & 26 & $\mathrm{E}$ & MA & Arch & WIMP & SS & - \\
\hline ChronoTwigger & (Ens et al., 2014) & 17 & $\mathrm{E}$ & ALL & Dev, Test & PostWIMP & WL & - \\
\hline SIFEI & (Kulesz et al., 2014) & 9 & $\mathrm{E}$ & ALL & N/A & Model & SS & $\checkmark$ \\
\hline AR map & (Dugerdil and Niculescu, 2014) & 5 & $\mathrm{~S}$ & DI & MTN & Model & SS & - \\
\hline regVIS & (Toprak et al., 2014) & 4 & $\mathrm{~S}$ & DI & N/A & Model & SS & $\checkmark$ \\
\hline Samoa & (Minelli and Lanza, 2013b) & 108 & $\mathrm{~S}$ & MA & N/A & Multivariate & SS & $\checkmark$ \\
\hline Azurite & (Yoon et al., 2013) & 57 & $\mathrm{E}$ & MA & Dev & Model & SS & $\checkmark$ \\
\hline SourceVis & (Anslow et al., 2013) & 36 & $\mathrm{E}$ & DI & Dev & PostWIMP & MT & - \\
\hline TraceDiff & (Trumper et al., 2013) & 31 & $\mathrm{~B}$ & DI & N/A & Multivariate & SS & - \\
\hline Performance evolution blueprint & (Sandoval Alcocer et al., 2013) & 31 & $\mathrm{E}$ & MA & N/A & Multivariate & SS & - \\
\hline SArF map & (Kobayashi et al., 2013) & 29 & $\mathrm{~S}$ & DI & Dev, User & Map & SS & - \\
\hline SyncTrace & (Karran et al., 2013) & 28 & $\mathrm{~B}$ & DI & N/A & Multivariate & SS & - \\
\hline Taggle & (Emerson et al., 2013) & 9 & $\mathrm{~S}$ & DI & Dev & Model & SS & $\checkmark$ \\
\hline SoftDynamik & (Grznár and Kapec, 2013) & 4 & $\mathrm{~B}$ & DI & Dev & Model & SS & - \\
\hline
\end{tabular}


Software Engineering Processes. We found that $48.58 \%$ of papers (i.e., 51 papers) relate to the design and implementation process. That is, we found that $22.86 \%$ of papers (i.e., 24 papers) related to visualizations proposed to support tasks of the maintenance process. We found that $21.91 \%$ of papers (i.e., 23 papers) relate to all processes in the software life-cycle, and that $6.67 \%$ of papers (i.e., 7 papers) relate to software testing process. We did not find visualizations proposed to support software requirements process, possibly due our employed method to collect primary studies. Of course, it is possible that software visualization papers that relate to the software requirements process are published, for instance, as short papers. In summary, we found that proposed software visualizations mostly support tasks involved with the design and implementation processes. Software maintenance is another frequent process in which we classified many proposed visualizations. We observe that aspects of evolution significantly relate to the design and implementation and maintenance process. In general, we observe there are multiple visualizations proposed to support various phases of software life-cycle. We found only a few visualizations that support tasks which involve the software testing process. We ask why there are only a few software visualizations especially designed to support software requirements engineering and software testing processes. Although we understand there is an intrinsic complexity in these subjects, we think these processes can represent an opportunity for future research. One reason that explains the many visualizations proposed to support all processes is that software development requires communicating several processes which, in some cases, may impose a challenge. In particular, the challenge of define a clear separation between processes, for which, visualization can be suitable by showing, for instance, an overview and progress of development. In addition, software is inherently tangible. We think that visualization can play an important role, in particular, to support the design and implementation processes. Indeed, we observe that the number of visualization that supports these processes is significant. Amongst papers that present visualization to support these processes, we found 12 available tools for design and implementation process. We also found 6 available visualization tools for maintenance and 7 tools for all software engineering processes. We only found 3 available visualization tools that support software testing tasks. We observe that these findings are consistent with that "developer" is the most frequent targeted software engineering role.

Software Engineering Roles. The envisioned user of a software visualization tool plays a particular role as a stakeholder in the life-cycle of a software system. We analyzed roles described in the reviewed papers to understand how well defined are these envisioned roles. We found that developer is the most frequently envisioned role of the users of software visualizations $(\times 12)$. Proposed software visualizations that target the developer audience, usually focus on supporting source code understanding. The developers role crosscuts the three aspects of software (i.e., structure, behavior, evolution) that are visualized. Developers need to understand the structure (e.g., to modify a legacy software system), the behavior (e.g., to identify issues with the execution of programs), and the evolution (e.g., to analyze how software projects evolve). However, we also observe that developers in real-world projects usually play more specific roles (e.g., analysts, testers). We believe that the high frequency of generic roles in the expected audience of software visualizations can be a symptom of a disconnect with the reality in real-world software projects (Merino et al., 2018b). We suggest researchers in the software visualization field to identify the specific context of the targeted users of their proposed visualizations. We also recommend to identify specific users roles by analyzing real-world software projects. Other less frequent roles $(\times 1-3)$ that play envisioned users of software visualizations are researchers, software engineers, users, practitioners, analysts and maintainers. Of course, developers can require multiple tools to understand a software system. We consider this to be an opportunity for developing such tools, which could be used for education and industrial work.

Information Visualization Techniques. We classified information visualization techniques that we found amongst the analyzed software visualization papers. In them, we found empirical methodologies $(58.10 \%$, i.e., 61 papers), applications $(29.53 \%$, i.e., 31 papers), interactions $(9.53 \%$, i.e., 10 papers), and system and frameworks $(2.86 \%$, i.e., 3 papers). we observe that empirical methodologies is the most frequent technique. We think that the needs of information visualization researchers and software engineers of developing empirical methods to support the design and implementation of novel software visualizations can explain it. Another reason that explains it is that ecosystems in which software systems execute have multiple characteristics which are, usually, hard to understand. To support such analysis, visualization can be helpful. The least frequent category is "system and framework", which corresponds to models and artifacts used to develop applications.

Mediums. The most frequent medium used to render software visualization is the standard computer screen ( $82.86 \%$, i.e., 87 papers), 3D environments $(7.62 \%$, i.e., 8 papers), wall displays (1.91\%, i.e., 2 papers), and multitouch tables $(0.96 \%$, i.e., 1 papers). We found that even though the majority of software visualizations ' are displayed on the standard computer screen, a few other media have been used as well. The analysis of the evolution of the various media used to display software visualizations shows a trend that suggests that in the future we could expect 


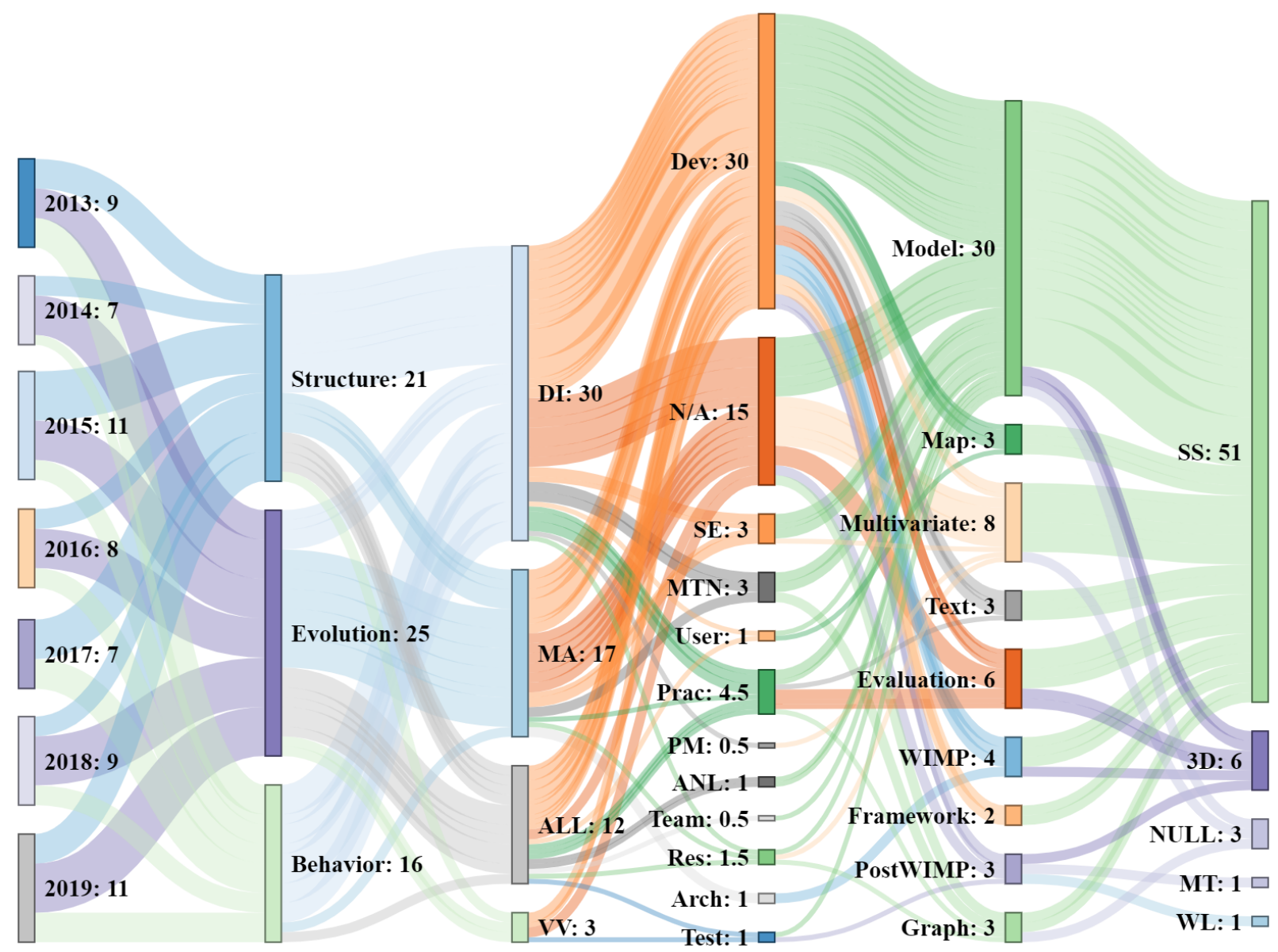

Fig. 5: A Sankey diagram that shows the distribution and relationships between software visualization papers published in 2013-2019. The levels in the diagram represent: year, software aspects, software processes, roles, information visualization techniques, and mediums, respectively.

that many proposed visualizations are going to be displayed in a medium other than the standard computer screen. For instance, we envision that with the rising of agile development, in which a team needs to discuss their work or present results to a steering committee in a meeting or war room, visualizations displayed in modern media will be used more frequently. Though we observe that these other modern media (e.g., virtual and augmented reality devices) require more support for users to interact and collaborate. For example, a visualization tool, such as ExplorViz, has been continuously improved. Recently, a report (Zirkelbach et al., 2019) describe hand gestures added to interact with the software visualization in VR.

Tools. we found multiple software visualization tools mentioned amongst papers, however, we observe that almost half of them are not available. We also observe that the average lifespan of software visualization tools is about 3.7 years (Merino et al., 2016). The reason for the short lifespan is one thing that should be considered for future research.

The Sankey diagram showed in Figure 5 presents the relationship amongst the characteristics of software visualizations, which are grouped into three aspects of software: Evolution, Structure, and Behavior. We observe that most of the visualizations support structural and behavioral aspects, which are often related to the design and implementation process. Also, the developer role is frequently envisioned as the target user of software visualizations. We observe that such role relates to the Model category using InfoVis techniques to support the design and implementation processes. Furthermore, most the standard computer screen is often the medium used to display software visualizations. We also notice that design and implementation processes heavily relate to the practitioner role who are associated to the evaluation in InfoVis the category. We conjecture that this could be explained as it is often necessary to measure and Evaluate the use of trainees in understanding software through software visualization tools. Software visualizations that support 
the analysis of evolution often support tasks involved in the maintenance process, though often the envisioned user is described as developer as opposed to maintainer, which could be explained in a lack of clear definition of the scope of the maintainer role. We observe that visualizations that support the software testing process focus on the evolution and structure aspects of systems. Similarly, the envisioned users of such visualizations are often developers and not testers.

In this paper, we report on the state-of-the-art of software visualization published between 2013-2019. We recommend to readers, possibly practitioners, interested in consulting software visualization published in previous years to access the software visualization blog https://softvis. wordpress.com/papers/overviews/.

\section{Conclusion}

In this paper, we provide an overview of the state-of-the-art of software visualization. We reviewed 105 software visualization papers published during the past six years. We concentrated on system papers as they often describe software visualization tools. System papers can be classified based on six aspects. From each paper, we extracted: (i) aspects of software visualization (i.e., structure, behavior, evolution), (ii) supported software engineering processes, (iii) roles of expected users, (iv) media used to display visualizations, (v) employed visualization techniques, and (vi) provide the software visualization tools list.

We found that about a third of the analyzed papers support software evolution tasks. We found the envisioned users of proposed software visualizations have multiple roles. We observe an increasing number of software visualizations proposed to support all software engineering processes, which could be due the fact that this process involves users that play multiple roles. The majority of software visualizations are displayed using a standard computer screen; however, we do observe that several approaches that support the analysis of the structure of systems are displayed in 3D environments with city metaphor. Interestingly, we found that the rate of proposed software visualizations that are displayed in a medium different than the computer screen is increasing consistently. When analyzing employed information visualization techniques, we observe that empirical methodologies are frequently used to support software engineering concerns. In addition, we also found that whereas the number of empirical studies conducted to evaluate software visualizations has gradually increased, software visualizations that support the analysis of multivariate data has increased as well. We found that the average lifespan of software visualization tools is 3.7 years. Amongst the identified software visualizations tools, we observe that only half of them are available.

We think our investigation can help developers to identify software visualization tools for concrete development tasks, for instance, a software development team could use storylines (Tang et al., 2018) to represent software development activity, as well as, a rich text visualization (Cao et al., 2010) for visualizing the software requirements.

In the future, we plan to expand our investigation by integrating the analysis of events and activities (Wu et al., 2018) and social media activity (Zhao et al., 2014; Cao et al., 2012). We also plan to investigate how visualizations displayed in immersive 3D environments such as head-mounted displays for virtual and augmented reality can help to increase the awareness of software requirements throughout the development life-cycle. We hope information visualization researchers, developers, and software engineering can use our classification to identify suitable visualization for their particular context in software projects.

Acknowledgements This work is supported by National Natural Science Foundation of China (61772463, 61772456, 61761136020). Merino acknowledges funding by the Deutsche Forschungsgemeinschaft (DFG, German Research Foundation) - Project-ID 251654672 - TRR 161. We thank Wilhelm Hasselbring, Michael Homer, Rainer Koschke, Roberto Minelli, Daniel Rozenberg, and Juraj Vincur, and for permitting us to reuse exemplary figures of their software visualizations.

\section{References}

Alexandru CV, Proksch S, Behnamghader P, Gall HC (2019) Evo-Clocks: Software evolution at a glance. In: IEEE Working Conference on Software Visualization (VISSOFT), IEEE, pp 12-22

Anquetil N, Etien A, Andreo G, Ducasse S (2019) Decomposing god classes at siemens. In: IEEE International Conference on Software Maintenance and Evolution (ICSME), IEEE, pp 169-180

Anslow C, Marshall S, Noble J, Biddle R (2013) SourceVis: Collaborative software visualization for co-located environments. In: IEEE Working Conference on Software Visualization (VISSOFT), IEEE, pp 1-10 
Barik T, Lubick K, Christie S, Murphy-Hill E (2014) How developers visualize compiler messages: A foundational approach to notification construction. In: IEEE Working Conference on Software Visualization (VISSOFT), IEEE, pp 87-96

Beck F, Burch M, Diehl S, Weiskopf D (2017a) A taxonomy and survey of dynamic graph visualization. Computer Graphics Forum 36(1):133-159

Beck F, Siddiqui HA, Bergel A, Weiskopf D (2017b) Method execution reports: Generating text and visualization to describe program behavior. In: IEEE Working Conference on Software Visualization (VISSOFT), IEEE, pp 1-10

Benomar O, Sahraoui H, Poulin P (2013) Visualizing software dynamicities with heat maps. In: IEEE Working Conference on Software Visualization (VISSOFT), IEEE, pp 1-10

Bergel A, Beck F (2017) Guest editorial of the special section on software visualization. Information and Software Technology 87:221-222

Burch M, Munz T, Beck F, Weiskopf D (2015) Visualizing work processes in software engineering with developer rivers. In: IEEE Working Conference on Software Visualization (VISSOFT), IEEE, pp 116-124

Cao N, Sun J, Lin YR, Gotz D, Liu S, Qu H (2010) Facetatlas: Multifaceted visualization for rich text corpora. IEEE Transactions on Visualization and Computer Graphics 16(6):1172-1181

Cao N, Lin YR, Sun X, Lazer D, Liu S, Qu H (2012) Whisper: Tracing the spatiotemporal process of information diffusion in real time. IEEE Transactions on Visualization and Computer Graphics 18(12):2649-2658

Clark B, Sharif B (2017) iTraceVis: Visualizing eye movement data within eclipse. In: IEEE Working Conference on Software Visualization (VISSOFT), IEEE, pp 22-32

Diehl S (2007) Software visualization: visualizing the structure, behaviour, and evolution of software. Springer Science $\&$ Business Media

Doray F, Dagenais M (2017) Diagnosing performance variations by comparing multi-level execution traces. IEEE Transactions on Parallel and Distributed Systems 28(2):462-474

Dugerdil P, Niculescu M (2014) Visualizing software structure understandability. In: Australian Software Engineering Conference (ASWEC), IEEE, pp 110-119

Duhoux B, Mens K, Dumas B (2018) Feature visualiser: an inspection tool for context-oriented programmers. In: International Workshop on Context-Oriented Programming: Advanced Modularity for Run-time Composition, ACM, pp 15-22

Emerson J, Churcher N, Deaker C (2013) From toy to tool: Extending tag clouds for software and information visualisation. In: Australian Software Engineering Conference (ASWEC), IEEE, pp 155-164

Ens B, Rea D, Shpaner R, Hemmati H, Young JE, Irani P (2014) ChronoTwigger: A visual analytics tool for understanding source and test co-evolution. In: IEEE Working Conference on Software Visualization (VISSOFT), IEEE, pp 117-126

Feiner J, Andrews K (2018) RepoVis: Visual overviews and full-text search in software repositories. In: IEEE Working Conference on Software Visualization (VISSOFT), IEEE, pp 1-11

Feist MD, Santos EA, Watts I, Hindle A (2016) Visualizing project evolution through abstract syntax tree analysis. In: IEEE Working Conference on Software Visualization (VISSOFT), IEEE, pp 11-20

Fittkau F, Krause A, Hasselbring W (2015a) Hierarchical software landscape visualization for system comprehension: A controlled experiment. In: IEEE Working Conference on Software Visualization (VISSOFT), IEEE, pp 36-45

Fittkau F, Zirkelbach C, Krause A, Hasselbring W (2015b) Explorviz. URL https://www.explorviz.net/, accessed February 25, 2020

Fronza I, Janes A, Sillitti A, Succi G, Trebeschi S (2013) Cooperation wordle using pre-attentive processing techniques. In: International Workshop on Cooperative and Human Aspects of Software Engineering (CHASE), IEEE, pp 57-64

Garzón-Rodriguez LP, Diosa HA, Rojas-Galeano S (2015) Deconstructing GAs into visual software components. In: Annual Conference on Genetic and Evolutionary Computation, ACM, pp 1125-1132

Geisler G (1998) Making information more accessible: A survey of information visualization applications and techniques. URL: http://www ils unc edu/ ${ }^{\sim}$ geisg/info/infovis/paper html

Gouveia C, Campos J, Abreu R (2013) Using HTML5 visualizations in software fault localization. In: IEEE Working Conference on Software Visualization (VISSOFT), IEEE, pp 1-10

Greene GJ, Fischer B (2015) Interactive tag cloud visualization of software version control repositories. In: IEEE Working Conference on Software Visualization (VISSOFT), IEEE, pp 56-65

Greene GJ, Esterhuizen M, Fischer B (2017) Visualizing and exploring software version control repositories using interactive tag clouds over formal concept lattices. Information and Software Technology 87:223-241 
Grznár F, Kapec P (2013) Visualizing dynamics of object oriented programs with time context. In: Spring Conference on Computer Graphics, ACM, pp 65-72

Hao R, Feng Y, Jones JA, Li Y, Chen Z (2019) CTRAS: Crowdsourced test report aggregation and summarization. In: IEEE/ACM International Conference on Software Engineering (ICSE), IEEE, pp 900-911

Hawes N, Marshall S, Anslow C (2015) CodeSurveyor: Mapping large-scale software to aid in code comprehension. In: IEEE Working Conference on Software Visualization (VISSOFT), IEEE, pp 96-105

Hollmann N, Hanenberg S (2017) An empirical study on the readability of regular expressions: Textual versus graphical. In: IEEE Working Conference on Software Visualization (VISSOFT), IEEE, pp 74-84

Homer M, Noble J (2014a) Combining tiled and textual views of code. In: IEEE Working Conference on Software Visualization (VISSOFT), IEEE, pp 1-10

Homer M, Noble J (2014b) Tiled grace. URL https://homepages.ecs.vuw. ac.nz/ mwh/minigrace/tiled/, accessed February 25, 2020

Isaacs KE, Gamblin T (2018) Preserving command line workflow for a package management system using ascii dag visualization. IEEE Transactions on Visualization and Computer Graphics 25(9):2804-2820

Isaacs KE, Bremer PT, Jusufi I, Gamblin T, Bhatele A, Schulz M, Hamann B (2014) Combing the communication hairball: Visualizing parallel execution traces using logical time. IEEE Transactions on Visualization and Computer Graphics 20(12):2349-2358

Karran B, Trumper J, Döllner J (2013) Synctrace: Visual thread-interplay analysis. In: IEEE Working Conference on Software Visualization (VISSOFT), IEEE, pp 1-10

Khaloo P, Maghoumi M, Taranta E, Bettner D, Laviola J (2017) Code Park: A new 3D code visualization tool. In: IEEE Working Conference on Software Visualization (VISSOFT), IEEE, pp 43-53

Khan T, Barthel H, Ebert A, Liggesmeyer P (2015) Visual analytics of software structure and metrics. In: IEEE Working Conference on Software Visualization (VISSOFT), IEEE, pp 16-25

Kienle HM, Muller HA (2007) Requirements of software visualization tools: A literature survey. In: IEEE International Workshop on Visualizing Software for Understanding and Analysis (VISSOFT), IEEE, pp 2-9

Kitchenham BA, Pfleeger SL, Pickard LM, Jones PW, Hoaglin DC, Emam KE, Rosenberg J (2002) Preliminary guidelines for empirical research in software engineering. IEEE Transactions on Software Engineering 22(8):721-734, DOI 10.1109/TSE.2002.1027796

Kobayashi K, Kamimura M, Yano K, Kato K, Matsuo A (2013) SArF map: Visualizing software architecture from feature and layer viewpoints. In: IEEE International Conference on Program Comprehension (ICPC), IEEE, pp $43-52$

Kulesz D, Scheurich J, Beck F (2014) Integrating anomaly diagnosis techniques into spreadsheet environments. In: IEEE Working Conference on Software Visualization (VISSOFT), IEEE, pp 11-19

Kumar S (2016) A review of recent trends and issues in visualization. International Journal on Computer Science and Engineering (IJCSE) 8(3):41-54

Lebeuf C, Voyloshnikova E, Herzig K, Storey MA (2018) Understanding, debugging, and optimizing distributed software builds: A design study. In: IEEE International Conference on Software Maintenance and Evolution (ICSME), IEEE, pp 496-507

Lessa IDM, Carneiro GDF, Monteiro MP, e Abreu FB (2015) A concern visualization approach for improving MAT$\mathrm{LAB}$ and octave program comprehension. In: Brazilian Symposium on Software Engineering (SBES), IEEE, pp 130-139

Liu S, Cui W, Wu Y, Liu M (2014) A survey on information visualization: recent advances and challenges. The Visual Computer 30(12):1373-1393

Liu S, Wang X, Liu M, Zhu J (2017) Towards better analysis of machine learning models: A visual analytics perspective. Visual Informatics 1(1):48-56

Maletic JI, Marcus A, Collard ML (2002) A task oriented view of software visualization. In: International Workshop on Visualizing Software for Understanding and Analysis (VISSOFT), IEEE, pp 32-40

Martinez J, Ziadi T, Mazo R, Bissyandé TF, Klein J, Le Traon Y (2014) Feature relations graphs: A visualisation paradigm for feature constraints in software product lines. In: IEEE Working Conference on Software Visualization (VISSOFT), IEEE, pp 50-59

Mattila AL, Ihantola P, Kilamo T, Luoto A, Nurminen M, Väätäjä H (2016) Software visualization today: Systematic literature review. In: International Academic Mindtrek Conference, ACM, pp 262-271

McNabb L, Laramee RS (2017) Survey of surveys (SoS)-mapping the landscape of survey papers in information visualization. Computer Graphics Forum 36(3):589-617 
Merino L, Ghafari M, Nierstrasz O (2016) Towards actionable visualisation in software development. In: IEEE Working Conference on Software Visualization (VISSOFT), IEEE, pp 61-70

Merino L, Ghafari M, Anslow C, Nierstrasz O (2018a) A systematic literature review of software visualization evaluation. Journal of Systems and Software 144:165-180

Merino L, Ghafari M, Nierstrasz O (2018b) Towards actionable visualization for software developers. Journal of Software: Evolution and Process 30(2):e1923

Merino L, Kozlova E, Nierstrasz O, Weiskopf D (2019) VISON: An ontology-based approach for software visualization tool discoverability. In: IEEE Working Conference on Software Visualization (VISSOFT), IEEE, pp 45-55

Middleton J, Murphy-Hill E (2016) Perquimans: A tool for visualizing patterns of spreadsheet function combinations. In: IEEE Working Conference on Software Visualization (VISSOFT), IEEE, pp 51-60

Minelli R, Lanza M (2013a) Samoa. URL http://samoa.inf .usi.ch/, accessed February 25, 2020

Minelli R, Lanza M (2013b) Software analytics for mobile applications-insights and lessons learned. In: European Conference on Software Maintenance and Reengineering (CSMR), IEEE, pp 144-153

Minelli R, Mocci A, Lanza M, Baracchi L (2014) Visualizing developer interactions. In: IEEE Working Conference on Software Visualization (VISSOFT), IEEE, pp 147-156

Molnar AJ (2015) JETracer a framework for Java GUI event tracing. In: International Conference on Evaluation of Novel Approaches to Software Engineering (ENASE), IEEE, pp 207-214

Mumtaz H, Latif S, Beck F, Weiskopf D (2019) Exploranative code quality documents. IEEE transactions on visualization and computer graphics 26(1):1129-1139

Munzner T (2008) Process and pitfalls in writing information visualization research papers. In: International Conference on Information Visualisation (IV), Springer, pp 134-153

Nguyen HTP, Bhatele A, Jain N, Kesavan S, Bhatia H, Gamblin T, Ma K, Bremer P (2019) Visualizing hierarchical performance profiles of parallel codes using callflow. IEEE Transactions on Visualization and Computer Graphics pp 1-1, DOI 10.1109/TVCG.2019.2953746

Ogami K, Kula RG, Hata H, Ishio T, Matsumoto K (2017) Using high-rising cities to visualize performance in realtime. In: IEEE Working Conference on Software Visualization (VISSOFT), IEEE, pp 33-42

Palepu VK, Jones JA (2015) Revealing runtime features and constituent behaviors within software. In: IEEE Working Conference on Software Visualization (VISSOFT), IEEE, pp 86-95

Perrie J, Xie J, Nayebi M, Fokaefs M, Lyons K, Stroulia E (2019) City on the river: visualizing temporal collaboration. In: International Conference on Computer Science and Software Engineering, pp 82-91

Porkoláb Z, Brunner T, Krupp D, Csordás M (2018) Codecompass: an open software comprehension framework for industrial usage. In: International Conference on Program Comprehension (ICPC), ACM, pp 361-369

Qian J, Chapin A, Papoutsaki A, Yang F, Nelissen K, Huang J (2018) Remotion: A motion-based capture and replay platform of mobile device interaction for remote usability testing. ACM on Interactive, Mobile, Wearable and Ubiquitous Technologies 2(2):77

Rabbi F, Lamo Y, Yu IC, Kristensen LM (2016) WebDPF: A web-based metamodelling and model transformation environment. In: International Conference on Model-Driven Engineering and Software Development (MODELSWARD), IEEE, pp 87-98

Rodrigues-Jr J, Zaina L, Oliveira M, Brandoli B, Traina A (2015) A survey on information visualization in light of vision and cognitive sciences. arXiv preprint arXiv:150507079

Rozenberg D, Beschastnikh I, Kosmale F, Poser V, Becker H, Palyart M, Murphy GC (2016) Comparing repositories visually with repograms. In: IEEE/ACM Working Conference on Mining Software Repositories (MSR), IEEE, pp $109-120$

Rüdel MO, Ganser J, Koschke R (2018) A controlled experiment on spatial orientation in VR-based software cities. In: IEEE Working Conference on Software Visualization (VISSOFT), IEEE, pp 21-31

Rufiange S, Melançon G (2014) Animatrix: A matrix-based visualization of software evolution. In: IEEE Working Conference on Software Visualization (VISSOFT), IEEE, pp 137-146

Sandoval Alcocer JP, Bergel A, Ducasse S, Denker M (2013) Performance Evolution Blueprint: Understanding the impact of software evolution on performance. In: IEEE Working Conference on Software Visualization (VISSOFT), IEEE, pp 1-9

Sandoval Alcocer JP, Beck F, Bergel A (2019) Performance Evolution Matrix: Visualizing performance variations along software versions. In: IEEE Working Conference on Software Visualization (VISSOFT), IEEE, pp 1-11

Scarle S, Walkinshaw N (2015) Visualising software as a particle system. In: IEEE Working Conference on Software Visualization (VISSOFT), IEEE, pp 66-75 
Schneider T, Tymchuk Y, Salgado R, Bergel A (2016) CuboidMatrix: Exploring dynamic structural connections in software components using space-time cube. In: IEEE Working Conference on Software Visualization (VISSOFT), IEEE, pp 116-125

Sensalire M, Ogao P, Telea A (2008) Classifying desirable features of software visualization tools for corrective maintenance. In: ACM Symposium on Software Visualization (SOFTVIS), ACM, pp 87-90

Shahin M, Liang P, Babar MA (2014) A systematic review of software architecture visualization techniques. Journal of Systems and Software 94:161-185

Sirkiä T (2018) Jsvee \& Kelmu: Creating and tailoring program animations for computing education. Journal of Software: Evolution and Process 30(2):e1924

Slater J, Anslow C, Dietrich J, Merino L (2019) CorpusVis-visualizing software metrics at scale. In: IEEE Working Conference on Software Visualization (VISSOFT), IEEE, pp 99-109

Sommerville I (2011) Software engineering. Person Education Ltd

Steinbeck M, Koschke R, Rüdel MO (2019) Movement patterns and trajectories in three-dimensional software visualization. In: International Working Conference on Source Code Analysis and Manipulation (SCAM), IEEE, pp $163-174$

Storey MAD, Čubranić D, German DM (2005) On the use of visualization to support awareness of human activities in software development: a survey and a framework. In: ACM Symposium on Software Visualization (SOFTVIS), ACM, pp 193-202

Sun GD, Wu YC, Liang RH, Liu SX (2013) A survey of visual analytics techniques and applications: State-of-the-art research and future challenges. Journal of Computer Science and Technology 28(5):852-867

Tang T, Rubab S, Lai J, Cui W, Yu L, Wu Y (2018) iStoryline: Effective convergence to hand-drawn storylines. IEEE Transactions on Visualization and Computer Graphics

Toosi AN, Son J, Buyya R (2018) CLOUDS-Pi: A low-cost Raspberry-Pi based micro data center for software-defined cloud computing. IEEE Cloud Computing 5(5):81-91

Toprak S, Wichmann A, Schupp S (2014) Lightweight structured visualization of assembler control flow based on regular expressions. In: IEEE Working Conference on Software Visualization (VISSOFT), IEEE, pp 97-106

Trumper J, Döllner J, Telea A (2013) Multiscale visual comparison of execution traces. In: IEEE International Conference on Program Comprehension (ICPC), IEEE, pp 53-62

Tymchuk Y, Merino L, Ghafari M, Nierstrasz O (2016) Walls, pillars and beams: A 3D decomposition of quality anomalies. In: IEEE Working Conference on Software Visualization (VISSOFT), IEEE, pp 126-135

Ulan M, Hönel S, Martins RM, Ericsson M, Löwe W, Wingkvist A, Kerren A (2018) Quality models inside out: Interactive visualization of software metrics by means of joint probabilities. In: IEEE Working Conference on Software Visualization (VISSOFT), IEEE, pp 65-75

Urli S, Bergel A, Blay-Fornarino M, Collet P, Mosser S (2015) A visual support for decomposing complex feature models. In: IEEE Working Conference on Software Visualization (VISSOFT), IEEE, pp 76-85

Vincur J, Navrat P, Polasek I (2017) VR City: Software analysis in virtual reality environment. In: IEEE International Conference on Software Quality, Reliability and Security Companion (QRS-C)

Wang Y, Weatherston J, Storey MA, German D (2019) CloneCompass: Visualizations for exploring assembly code clone ecosystems. In: IEEE Working Conference on Software Visualization (VISSOFT), IEEE, pp 88-98

Wilde E, German D (2018) Merge-Tree: Visualizing the integration of commits into linux. Journal of Software: Evolution and Process 30(2):e1936

Wilhelm A, Cakaric F, Schuele T, Gerndt M (2018) Tool-based interactive software parallelization: a case study. In: IEEE/ACM International Conference on Software Engineering: Software Engineering in Practice Track (ICSESEIP), IEEE, pp 115-123

Williams K, Bigelow A, Isaacs K (2019) Visualizing a moving target: A design study on task parallel programs in the presence of evolving data and concerns. IEEE Transactions on Visualization and Computer Graphics 26(1):11181128

Wu Y, Chen Z, Sun G, Xie X, Cao N, Liu S, Cui W (2018) Streamexplorer: a multi-stage system for visually exploring events in social streams. IEEE Transactions on Visualization and Computer Graphics 24(10):2758-2772

Xu K, Wang Y, Yang L, Wang Y, Qiao B, Qin S, Xu Y, Zhang H, Qu H (2019) Clouddet: Interactive visual analysis of anomalous performances in cloud computing systems. IEEE transactions on visualization and computer graphics 26(1):1107-1117

Yi JS, ah Kang Y, Stasko J (2007) Toward a deeper understanding of the role of interaction in information visualization. IEEE Transactions on Visualization and Computer Graphics 13(6):1224-1231 
Yoon Y, Myers BA, Koo S (2013) Visualization of fine-grained code change history. In: IEEE Symposium on Visual Languages and Human-Centric Computing (VL/HCC), IEEE, pp 119-126

Zhao J, Cao N, Wen Z, Song Y, Lin YR, Collins C (2014) \#FluxFlow: Visual analysis of anomalous information spreading on social media. IEEE Transactions on Visualization and Computer Graphics 20(12):1773-1782

Zhu J, Alderfer K, Furqan A, Nebolsky J, Char B, Smith B, Villareale J, Ontañón S (2019) Programming in game space: how to represent parallel programming concepts in an educational game. In: International Conference on the Foundations of Digital Games, pp 1-10

Zhu X, Nacenta MA, Akgun O, Nightingale P (2019) How people visually represent discrete constraint problems. IEEE Transactions on Visualization and Computer Graphics pp 1-1, DOI 10.1109/TVCG.2019.2895085

Zirkelbach C, Krause A, Hasselbring W (2019) Hands-on: experiencing software architecture in virtual reality. Tech. rep., Department of Computer Science, Kiel University, Germany 